\title{
The Role of Endoscopic Ultrasound-guided Drainage for Autoimmune Pancreatitis-associated Pancreatic Cysts: A Report of Five Cases and a Literature Review
}

\author{
Kenjiro Yamamoto ${ }^{1}$, Takao Itoi ${ }^{1}$, Atsushi Sofuni ${ }^{1}$, Takayoshi Tsuchiya ${ }^{1}$, Shujiro Tsuji ${ }^{1}$, \\ Reina Tanaka ${ }^{1}$, Ryosuke Tonozuka ${ }^{1}$, Mitsuyoshi Honjo ${ }^{1}$, Shuntaro Mukai ${ }^{1}$, Kentaro Kamada ${ }^{1}$, \\ Mitsuru Fujita ${ }^{1}$, Yasutsugu Asai ${ }^{1}$, Yukitoshi Matsunami ${ }^{1}$ and Yuichi Nagakawa ${ }^{2}$
}

\begin{abstract}
:
Objective Autoimmune pancreatitis (AIP) has been recognized as a benign disease, which that shows a prompt response to corticosteroid treatment (CST). It was previously believed to not be associated with cyst formation; however, a few cases of AIP-associated pancreatic cyst (PC) have been reported. Some cases were reported to have been effectively treated by CST, while others were refractory to CST. Many of the patients received interventional treatment. Until now, there has been no consensus on the therapeutic strategies for AIP-associated PC. The aim of the present study is to describe a therapeutic strategy for this condition.

Methods We conducted a retrospective study of 5 cases of AIP-associated PC that were treated by endoscopic ultrasonography-guided pancreatic fluid collection drainage (ESPD) or CST at Tokyo Medical University Hospital between March 2012 and October 2016, analyzed the therapeutic outcomes, and performed a literature review.

Results The initial treatments included CST $(n=2)$ and ESPD $(n=3)$. All of the PCs disappeared after treatment In 1 of the patients who received CST case and 3 of the patients who received ESPD; however, the PC did not disappear in one of the patients who received CST (corticosteroid maintenance therapy), even after the dose of corticosteroids was increased; ESPD was eventually performed and the PC disappeared. There were no procedure-related complaints.

Conclusion We propose that CST be administered as the first-line treatment for AIP-associated PC, particularly in cases of PC without a history of CST. However, ESPD can be applied to treat cases of corticosteroid refractory PC.
\end{abstract}

Key words: autoimmune pancreatitis, pancreatic cyst, corticosteroid treatment, endoscopic ultrasonographyguided pancreatic fluid collection drainage

(Intern Med 57: 1523-1531, 2018)

(DOI: 10.2169/internalmedicine.9779-17)

\section{Introduction}

Autoimmune pancreatitis (AIP) is a benign disease that was first reported in 1961. Since the first report, the clinical conditions associated with AIP have gradually been elucidated. Currently, AIP is thought to occur as part of a systemic disease produced by IgG4-positive lymphoplasmacytic infiltration of the involved organs in association with an elevation in the blood level of IgG4 (type 1), or as a primary disease of the pancreas (type 2) $(1,2)$. Recently, the International Association of Pancreatology presented a set of diagnostic criteria for AIP $(3,4)$. Importantly, it was previously thought that AIP was not associated with the development of pancreatic cysts (5). However, there have been a few reports of AIP with an accompanying pancreatic cyst (6-14). More-

${ }^{1}$ Department of Gastroenterology and Hepatology, Tokyo Medical University, Japan and ${ }^{2}$ Department of Third, Tokyo Medical University, Japan Received: July 7, 2017; Accepted: September 26, 2017; Advance Publication by J-STAGE: February 9, 2018

Correspondence to Dr. Takao Itoi, itoi@tokyo-med.ac.jp 

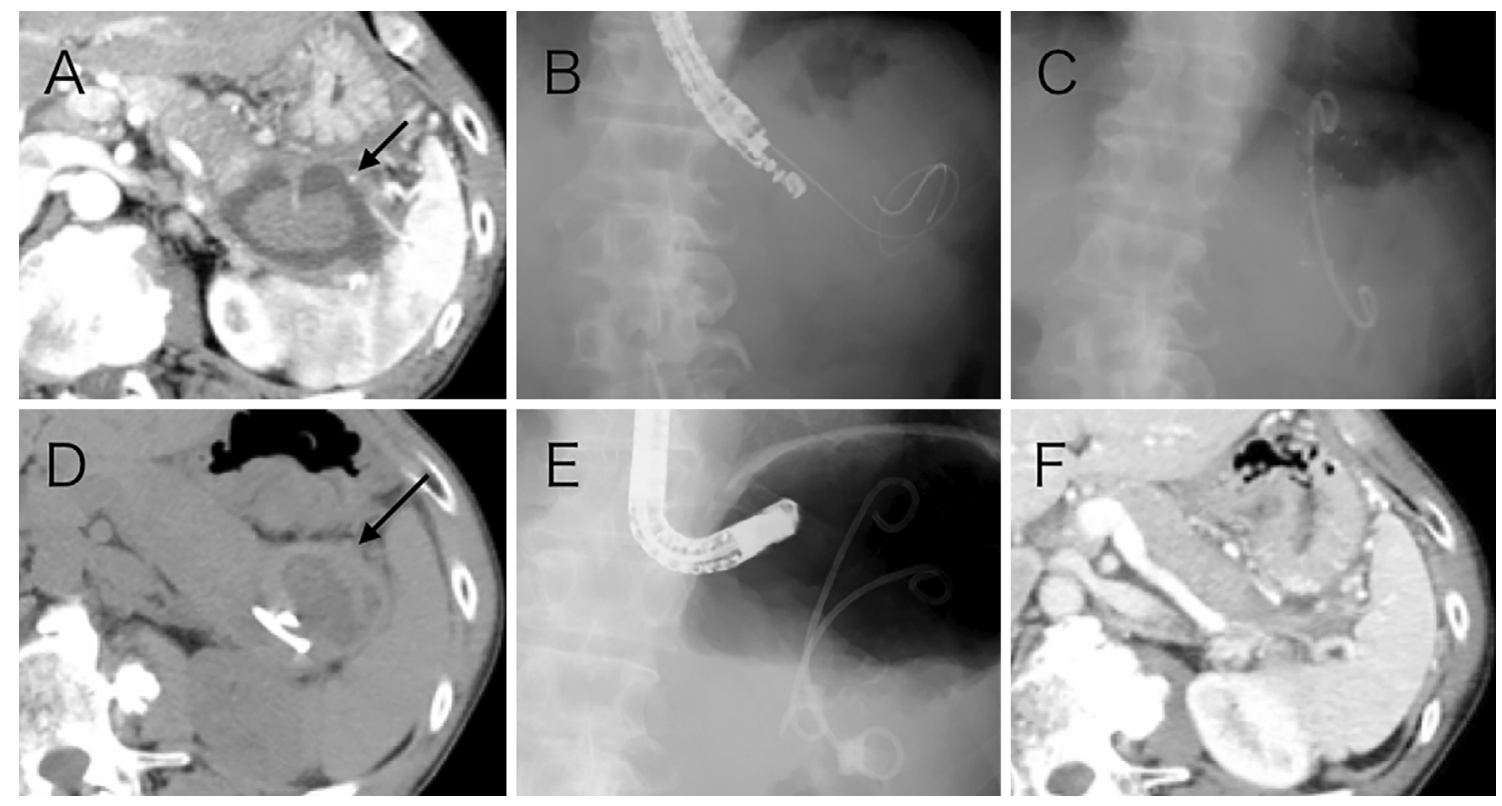

Figure 1. (A) Abdominal CT showed the diffuse enlargement of the pancreas and a cyst (arrow) of $60 \mathrm{~mm}$ diameter with hemorrhage in the pancreatic tail. (B, C) ESPD was performed by placing a BFMS and a plastic stent. (D) CT showed a reduction in the size of the pancreatic cyst (arrow) to 40 mm diameter. (E) Another 7-Fr double pigtail stent was re-inserted to the pancreatic cyst through the fistula. (F) CT performed 1 year and 4 months after ESPD showed the complete disappearance of the pancreatic cyst. ESPD: endoscopic ultrasonography-guided pancreatic fluid collection drainage

over, some reports have shown corticosteroid treatment (CST) to be highly effective in treating the accompanying pancreatic cyst $(6,10,15-19)$. However, surgical or interventional radiology treatments have occasionally been selected $(7,9,18,20,21)$.

To date, there has been no consensus on the therapeutic strategy for pancreatic cysts (PCs) that accompany AIP. We herein present 5 cases of AIP-associated PC that were treated by endoscopic ultrasonography-guided pancreatic fluid collection drainage (ESPD) or CST, and describe the therapeutic strategy. We also perform a literature review.

\section{Materials and Methods}

\section{Case 1}

The patient was a 61-year-old man who had consulted another hospital two years previously with a complaint of abdominal pain. The patient was diagnosed with chronic pancreatitis complicated by a pancreatic tail cyst. The patient was referred to our hospital because of the repeated acute exacerbation of chronic pancreatitis. Abdominal computed tomography (CT) revealed a "sausage-like" diffuse enlargement of the pancreas and a cyst of $60 \mathrm{~mm}$ diameter with hemorrhage in the pancreatic tail (Fig. 1A).

The laboratory data revealed a significantly increased serum IgG4 level (1,050 mg/dL; normal range, 4.8-105 mg/ $\mathrm{dL})$. Thus, he was diagnosed with AIP complicated by a PC in accordance with the Clinical Diagnostic Criteria for Autoimmune Pancreatitis 2011 (22). ESPD was performed by placing a biflanged metal stent (BFMS) (AXIOS stent, Boston Scientific, Natick, USA) and a 7-Fr double pigtail stent (Fig. 1B and C). After the placement of the stents, dark red fluid flowed out through the stent and the patient's symptoms disappeared immediately. At 6 days after ESPD, follow-up CT showed a reduction in the diameter of the pancreatic cyst to $40 \mathrm{~mm}$ (Fig. 1D). The metallic stent undeployed into the stomach; thus, another 7-Fr double pigtail stent was re-inserted (Fig. 1E). The patient was discharged from our hospital on the tenth day after ESPD, and was followed up as an outpatient without CST.

A CT scan performed 1 year and 4 months later showed the complete disappearance of the cyst (Fig. 1F); thereafter, the plastic stent was endoscopically removed. Cystic recurrence was not observed for more than 2 years after ESPD without CST.

\section{Case 2}

The patient was a 54-year-old man consulted our hospital with complaints of epigastric pain and vomiting after meals. Abdominal CT on admission revealed diffuse enlargement of the pancreas and a cyst of $100 \mathrm{~mm}$ diameter with hemorrhage in the pancreatic tail (Fig. 2A). The laboratory data revealed significantly increased levels of serum C-reactive protein (CRP level; $6.5 \mathrm{mg} / \mathrm{dL}$ ), serum amylase (388 U/L; normal range, 40-122 U/L), and serum IgG4 (290 mg/dL).

Based on these findings and her clinical course, the patient was diagnosed as having AIP complicated by a pancreatic cyst, and thus he was admitted to our hospital. Because of his prolonged symptoms, ESPD was performed by plac- 


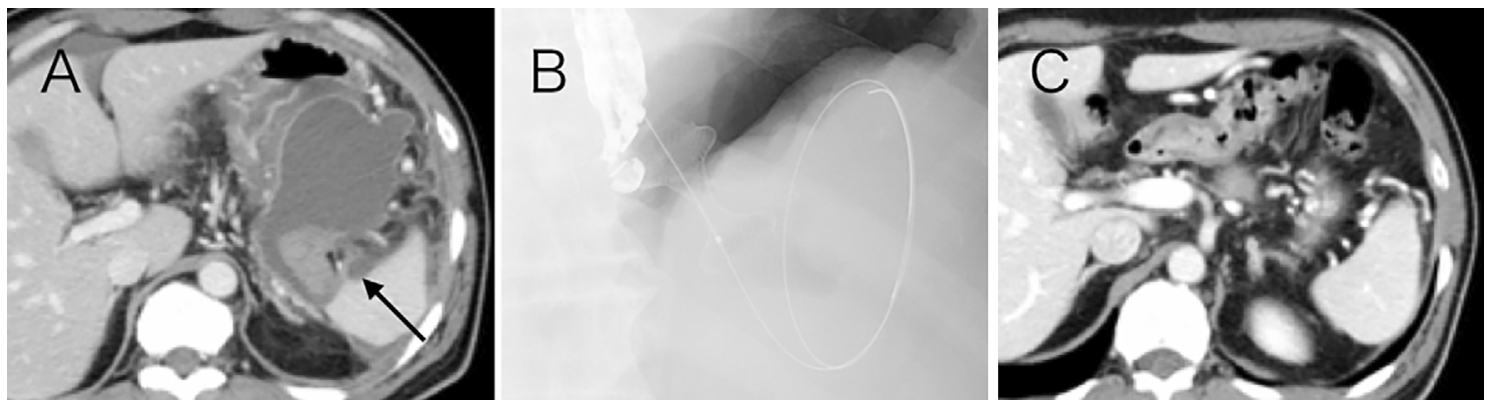

Figure 2. (A) CT showed a cyst (arrow) of $100 \mathrm{~mm}$ diameter with hemorrhage in the pancreatic tail. (B) ESPD was performed by placing a BFMS. (C) CT showed the absence of the pancreatic cyst. ESPD: endoscopic ultrasonography-guided pancreatic fluid collection drainage, BFMS: biflanged metal stent

ing a BFMS (AXIOS stent, Boston Scientific) (Fig. 2B). After the placement of the stent, dark red fluid flowed out through the stent and his symptoms disappeared immediately. Magnetic resonance imaging performed 10 days after ESPD showed a reduction in the size of the cyst and the patient was discharged 13 days after ESPD. The patient was started on prednisolone at a dose of $40 \mathrm{mg}$, daily, for 2 weeks. Thereafter, the dose was tapered by $5 \mathrm{mg}$ per week. The patient's serum IgG4 level decreased 11 months after ESPD, and follow-up CT showed the absence of both the cyst and the pancreatic enlargement (Fig. 2C).

\section{Case 3}

The patient was a 63-year-old man who had consulted our hospital 2 years previously with complaints of nausea and upper gastric pain. Abdominal CT revealed the diffuse enlargement of the pancreas and a 40-mm-diameter cyst in the pancreatic tail. The laboratory data revealed a significantly increased serum IgG4 level (706 mg/dL).

The patient was diagnosed with AIP complicated by a pancreatic cyst. He was treated with prednisolone at a dose of $40 \mathrm{mg}$, daily, for 2 weeks. This resulted in the resolution of the abdominal pain and the swelling of the pancreas; however, the cyst remained in the pancreatic tail. The dose was then tapered by $5 \mathrm{mg}$ per week. Two years after starting CST, and while the patient was being treated with prednisolone $(5 \mathrm{mg} /$ day), he visited our hospital with a complaint of left upper abdominal pain. CT revealed the enlargement of the pancreatic cyst to $60 \mathrm{~mm}$ diameter (Fig. 3A) and the presence of a lower bile duct stricture. He was diagnosed with recurrent AIP. However, as the bile duct stricture showed no obstructive jaundice and cholangitis, he was treated with a higher dose of prednisolone (25 mg, daily) without biliary drainage.

However, 2 weeks after the treatment, his left upper abdominal pain worsened and his laboratory data showed elevated levels of inflammatory markers, which led to the suspicion of an infected pancreatic cyst. We then performed ESPD for the pancreatic cyst using a 7-Fr double pigtail stent (Fig. 3B and C). After the placement of the stent, dark red fluid flowed out through the stent. After ESPD and in- travenous antibiotic therapy, the patient's symptom disappeared and he was discharged 7 days after ESPD. The cyst completely disappeared at 3 months after the ESPD (Fig. 3D). There was no recurrence for 6 months, and the patient was treated with prednisolone ( $5 \mathrm{mg}$, daily).

\section{Case 4}

The patient was a 64-year-old man who had visited another hospital one year previously with recurrent acute pancreatitis that had improved with conservative therapy. One month previously, he had visited our hospital with a complaint of left upper abdominal pain. CT showed the diffuse enlargement of the pancreas and a cyst of $130 \mathrm{~mm}$ diameter with hemorrhage in the pancreatic tail (Fig. 4A). Moreover, the patient's laboratory data revealed a significantly increased serum IgG4 level (706 mg/dL). The patient was diagnosed with AIP complicated by a pancreatic cyst and was referred to our hospital.

On the fourth day after admission, we performed ESPD for the pancreatic cyst by placing a BFMS (HANARO stent, MI Tech, Seoul, Korea) (Fig. 4B). After the placement of the stent, dark red fluid flowed out through the stent and his symptoms disappeared immediately. The patient was discharged from our hospital 13 days after ESPD. Follow-up CT at 2 months after ESPD showed a reduction in the size of the cyst, which prompted the endoscopic removal of the metallic stent and the placement of a 7-Fr double pigtail stent in the cyst. CST had been planned, however, at 6 months after ESPD he presented to our hospital with complaints of fever and abdominal pain. CT showed a large pancreatic cyst beside the left liver lobe and multiple small lesions in the liver (Fig. 4C and D). Recurrent PC and multiple liver abscesses were diagnosed. ESPD was performed for the recurrent cyst by placing a BFMS (HANARO stent, MI Tech) (Fig. 4E). After stent placement, dark red fluid flowed out through the stent and his symptoms disappeared immediately. Four days after ESPD, follow-up CT revealed a reduction in the size of the cyst located beside the hepatic left lobe. As the pancreatic tail cyst remained small, we placed a 5-Fr nasocystic catheter on the side of the plastic stent for the cyst. The multiple liver abscesses were treated 


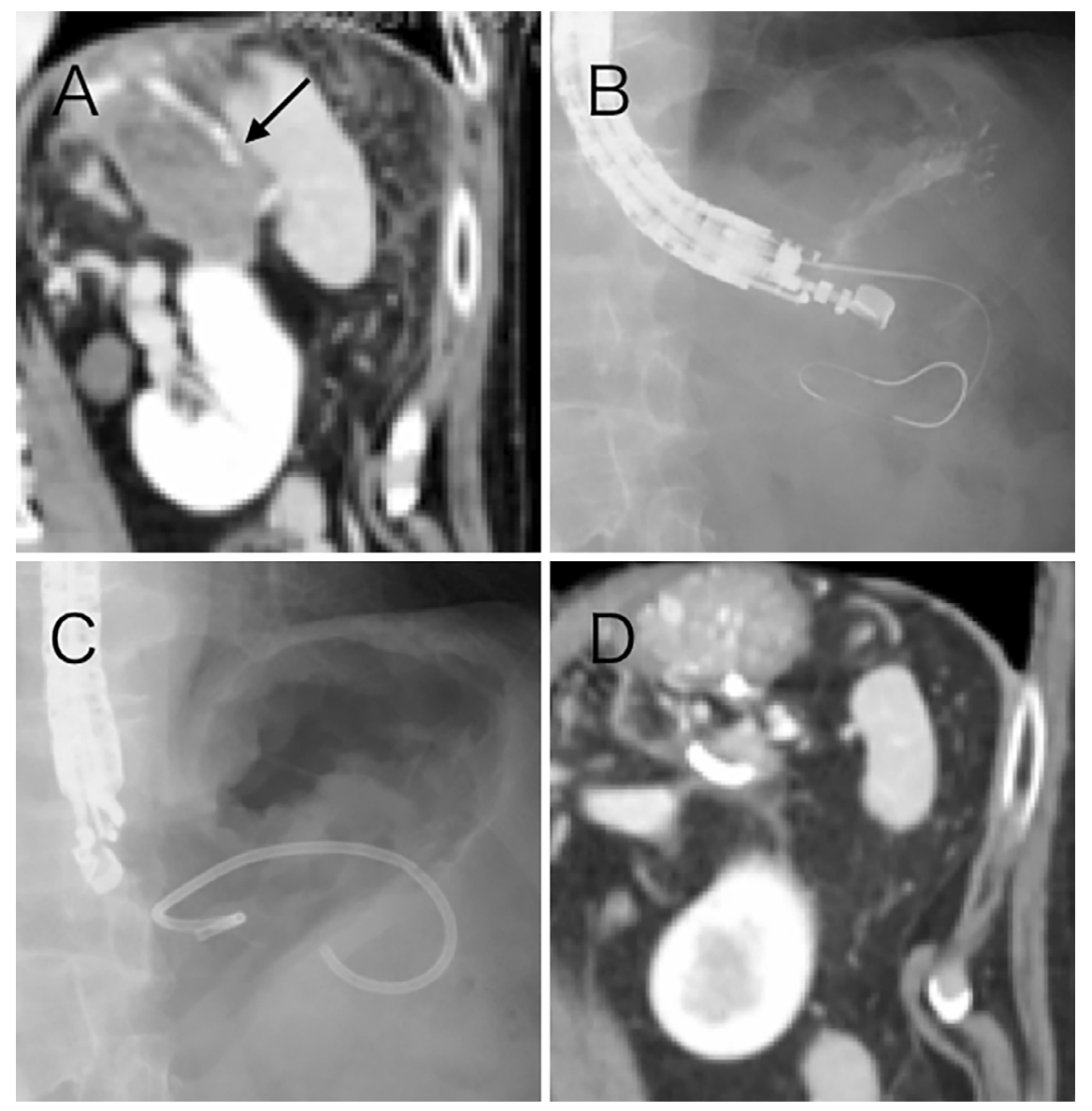

Figure 3. (A) CT showed the enlargement of the pancreatic cyst to $60 \mathrm{~mm}$ diameter (arrow). (B, C) ESPD was performed by placing a plastic stent. (D) The pancreatic cyst had completely disappeared at 3 months after ESPD. ESPD: endoscopic ultrasonography-guided pancreatic fluid collection drainage
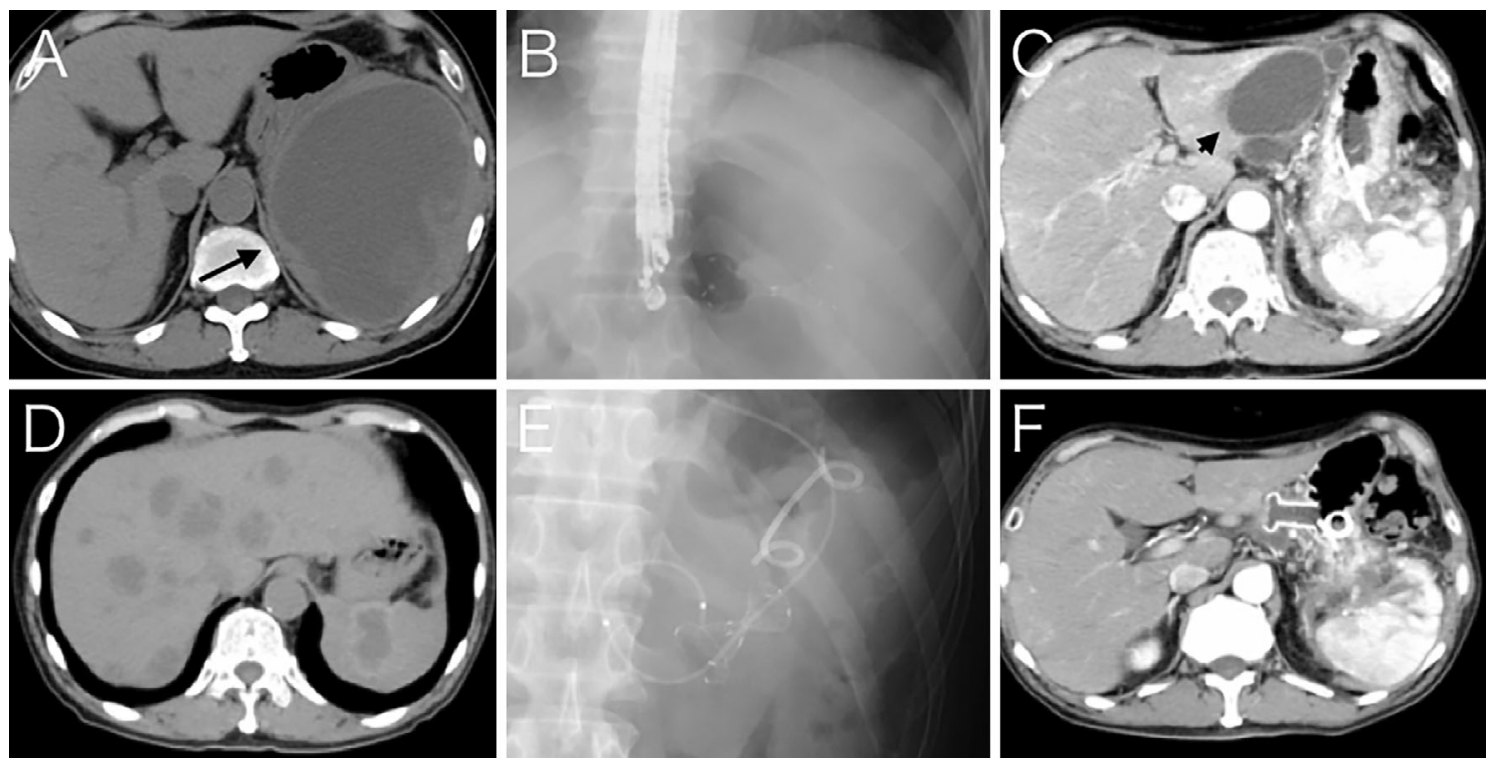

Figure 4. (A) CT showed a pancreatic cyst of $130 \mathrm{~mm}$ diameter with hemorrhage in the pancreatic tail (arrow). (B) ESPD was performed by placing a BFMS. (C, D) CT showed a large pancreatic cyst beside the left liver lobe (arrowhead) and multiple small lesions in the liver. (E) ESPD was performed for the recurrent pancreatic cyst by placing a BFMS. (F) CT at 1 month after ESPD showed the absence of both the pancreatic cyst and the multiple liver abscesses. ESPD: endoscopic ultrasonography-guided pancreatic fluid collection drainage, BFMS: biflanged metal stent 


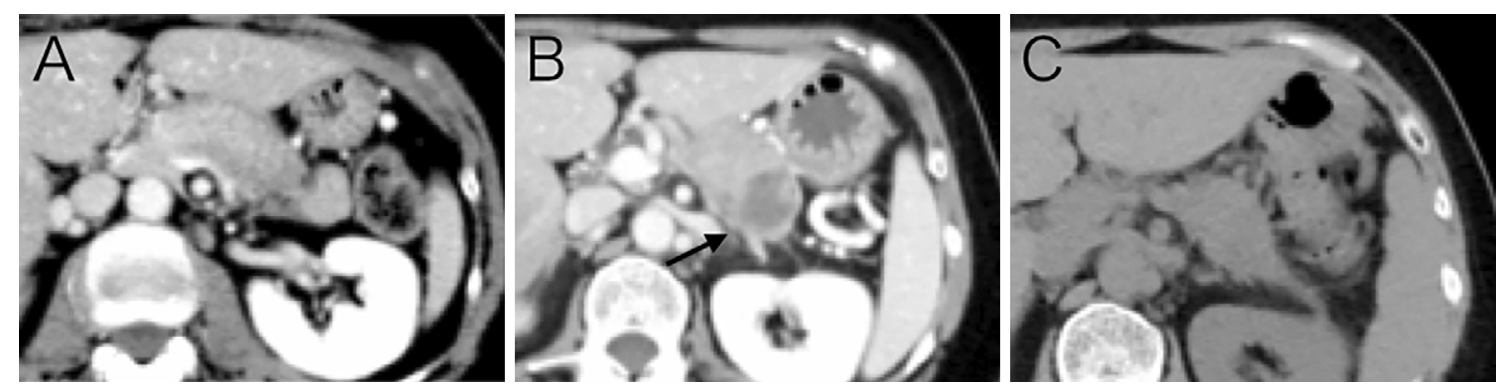

Figure 5. (A) CT showed diffuse pancreatic swelling. (B) CT showed a 25-mm-diameter cyst in the pancreatic tail (arrow). (C) CT showed the complete disappearance of the pancreatic cyst with the resolution of the pancreatic swelling.

with antibiotics for 4 weeks. Follow-up CT performed one month after ESPD revealed no cysts or multiple liver abscesses (Fig. 4F) and the patient was discharged. At two months after the second ESPD procedure, there was no evidence of recurrence of the PC or liver abscesses, and CST was planned.

\section{Case 5}

The patient was a 56-year-old woman who had been referred to our hospital with suspected AIP six months previously. Although her serum IgG4 level was normal, abdominal CT showed diffuse pancreatic swelling (Fig. 5A). However, IgG4-positive lymphoplasmacytic infiltration could not be detected by endoscopic ultrasound-fine needle aspiration. Moreover, she refused to undergo endoscopic retrograde pancreatography; thus, she was followed up as an outpatient without a diagnosis of AIP.

Two days prior to her diagnosis, she visited the presented emergently with nausea and epigastric pain, which had started 2 days previously. CT showed diffuse pancreatic swelling with delayed enhancement, similar to the initial CT findings and a cyst of $25 \mathrm{~mm}$ in diameter in the pancreatic tail (Fig. 5B). Her serum IgG4 level was high (184 mg/dL). She was therefore diagnosed as having AIP with a pancreatic cyst.

The patient was admitted to our hospital for closer examination and treatment. She was treated with $40 \mathrm{mg}$ of prednisolone daily for 2 weeks, after which the dose was tapered by $5 \mathrm{mg}$ per week. At three weeks after starting CST, abdominal CT showed the complete disappearance of the cyst with the resolution of pancreatic swelling (Fig. 5C). The patient was treated with corticosteroid maintenance therapy [prednisolone $(5 \mathrm{mg} / \mathrm{day})]$, and there was no recurrence of $\mathrm{PC}$ at 10 months after the onset of disease.

\section{Discussion}

AIP has been recognized as a benign disease that promptly responds to CST. It was previously believed that it did not progress to chronic pancreatitis associated with cyst formation or pancreatolithiasis (5). However, a few cases AIP-associated PC have been reported since
$2003(6-15,23)$.

Some reports have shown that CST was effective in most cases $(6,10,15-19)$. However, corticosteroid refractory PCs have also been reported $(18,24)$. Furthermore, surgical therapy or interventional radiology treatment has occasionally been selected without $\operatorname{CST}(7,9,18,20,21)$. Thus, there is still no consensus on the therapeutic strategy for AIPassociated PC.

There are currently 2 opinions regarding the mechanism of pancreatic cyst formation in AIP. Kubota et al. suggested that PC formation in AIP is associated with left-sided portal hypertension, which is closely associated with the inflammatory process of AIP, which is in a highly active state due to compression by the swollen pancreatic parenchyma and stenosis or occlusion of the peripancreas vascular involvement (18). On the other hand, Matsubayashi et al. inferred that severe stricture of the main pancreatic duct causes the inflammatory process leading to stasis of the pancreatic juice upstream and resulting in PC formation (19).

We hypothesize that PCs occur in AIP due to the pancreatic juice stasis caused by severe stricture of the pancreatic duct, as Matsubayashi et al. described. However, we believe that there are 2 processes underlying the development of severe stricture of the pancreatic duct, namely, the inflammation process and the fibrosis process, progressing to chronic pancreatitis.

The highly active inflammation process mainly occurs in the early stage of AIP. Spontaneous remission might occur, or a favorable response to CST might lead to the amelioration of the narrowing of the pancreatic duct and release the pancreatic juice. These induce the resolution of the pancreatic duct and the disappearance of the PC.

On the other hand, AIP has the potential to progress - through fibrosis - to chronic pancreatitis. Murayama et al. reported that the narrowing of both Wirsung's and Santorini's ducts in AIP might lead to pancreatic juice stasis and stone development, resulting in progression to a long-term chronic state (25). They also reported that relapse was more frequently observed in cases that progressed to chronic pancreatitis. Kamisawa et al. reported that $92 \%$ of AIP patients relapsed within 3 years from the start of CST (26), suggesting that AIP progressed-due to fibrosis-to chronic pancreati- 


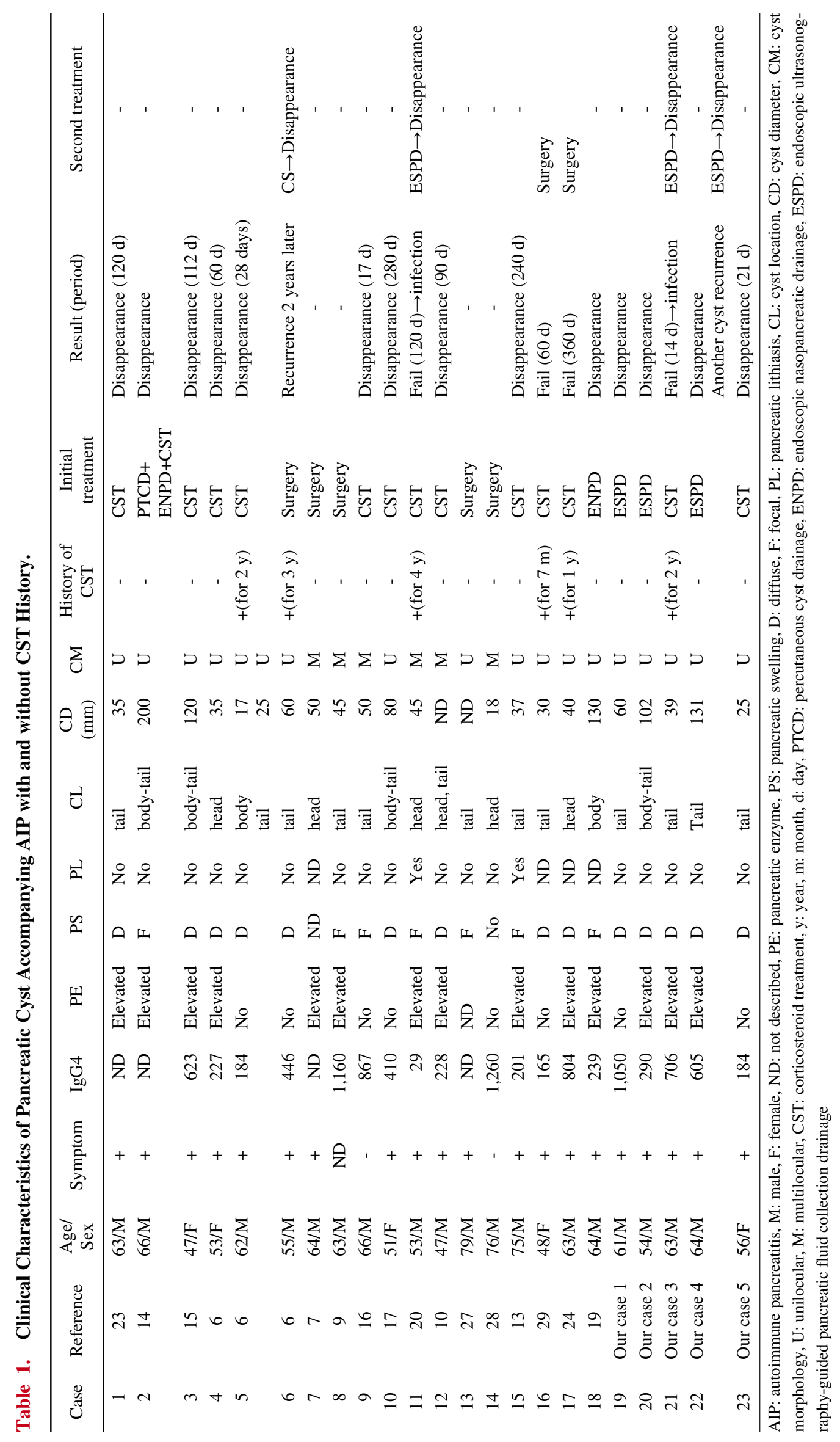


Table 2. Result of CST for Pancreatic Cyst Accompanying AIP with and without CST History.

\begin{tabular}{|c|c|c|}
\hline & Without CST history & With CST history \\
\hline Number of patients & 8 & 5 \\
\hline The mean serum IgG4 level (mg/dL) & 359 & 430 \\
\hline Pancreatic swelling (Diffuse/Focal) & $6 / 2$ & $4 / 1$ \\
\hline Pancreatic lithiasis (+/-/ND) & $1 / 7 / 0$ & $1 / 2 / 2$ \\
\hline Cyst size (mm) & $25-120$ & $17-60$ \\
\hline Cyst morphology (Unilocular/multilocular) & $7 / 2$ & $4 / 1$ \\
\hline The time of corticosteroid maintenance therapy & - & 7 months-2 years \\
\hline \multicolumn{3}{|l|}{$<$ Result of treatment $>$} \\
\hline Initial treatment by CST & All Disappearance & 4 Failure ( 2 infected cysts) \\
\hline [The period of treatment (day)] & {$[17-280]$} & {$[14-360]$} \\
\hline Second treatment & & All Disappearance (2 ESPD, 2 Surgery) \\
\hline
\end{tabular}

CST: corticosteroid treatment, AIP: autoimmune pancreatitis, ND: not described, ESPD: endoscopic ultrasonography-guided pancreatic fluid collection drainage

tis during CST.

The clinical characteristics of the reported cases with and without a history of CST are summarized in Table 1. The initial treatments were as follows: CST alone $(n=13)$, surgery alone $(n=5)$, ESPD alone $(n=3)$, endoscopic nasopancreatic drainage (ENPD) alone $(n=1)$, and percutaneous cyst drainage $(\mathrm{PTCD})+\mathrm{ENPD}+\mathrm{CST}(\mathrm{n}=1)$. Table 2 shows the results of CST for PCs accompanying AIP with and without a history of CST. Interestingly, in 8 cases without a history of CST, all PCs disappeared after CST (cases 1, 3, 4, 9, 10, 12, 15 , and 23$)(6,10,13,15-17,23)$. On the other hand, in 5 cases with a history of CST, a cyst appeared during corticosteroid maintenance therapy (cases 5, 6, 11, 16, 17, and $21)(6,20,24,29)$. Although the 2 small cysts (diameters, $17 \mathrm{~mm}$ and $25 \mathrm{~mm}$ ) in case 5 (6) disappeared after increasing the dose of corticosteroids, the cysts in 4 cases (cases $11,16,17$, and 21) $(20,24,29)$ did not disappear after an increase in the dose of corticosteroids; thus, these cysts are referred to as corticosteroid refractory cysts. The second treatments of these cases included surgical treatment in two cases (cases 16 and 17) $(24,29)$ and ESPD in two cases (cases 11 and 21) (20). One patient (case 6) (6) underwent direct surgical treatment; however, the cyst reappeared 2 years later but disappeared after the dose of corticosteroids was increased; thus, all of the patients with corticosteroid refractory PCs received corticosteroid maintenance treatment. We suggest that in patients with corticosteroid refractory PCs, irreversible chronic fibrotic changes had occurred in the pancreatic duct stricture; thus, CST alone is less likely to be effective.

Although CST was associated with a reduction of the size of the PCs in all of the patients without a history of CST, their symptoms disappeared immediately after CST. In contrast, in the cases of corticosteroid refractory PC, all of the cysts increased in size and 2 cysts were complicated by infection after the dose of corticosteroids was increased. Meanwhile, there were no differences in other factors such as the serum IgG4 level, pancreatic swelling, pancreatic lith- iasis, and cyst morphology in PCs accompanying AIP with and without a history of CST, and larger cysts were not always refractory to CST.

Four refractory cases received interventional treatment, namely, surgical treatment (cases 16 and 17) $(24,29)$ and ESPD (cases 11 and 21) (20). One case (case 6) (6) received direct surgical treatment; however, the cyst reappeared 2 years later but disappeared after an increase in the dose of corticosteroids; thus, all of the cases of corticosteroid refractory PC received corticosteroid maintenance treatment. We hypothesize that irreversible chronic fibrotic changes occur in the pancreatic duct stricture of patients with corticosteroid-refractory PCs; thus, the administration of CST alone would be less likely to be effective for such patients.

Among the 5 cases in which surgery was selected as the initial treatment (cases 6, 7, 8, 13, and 14) $(6,7,9,27,28)$, 2 cases were suspected to involve a cystic neoplasm (cases 7 and 14$)(7,28)$, in 1 case, the patient's physical condition did not improve with conservative medical treatment without CST (case 8) (9), in 1 case, the patient showed an accompanying splenic infarction (case 13) (27), and in 1 case, a cyst appeared during corticosteroid maintenance therapy (case 6) (6). All of the patients who received interventional radiology treatments, which included ESPD alone (cases 19, 20, and 22), ENPD alone (case 18) (19), and PTCD+ENPD+ CST (case 2) (14), were effectively treated.

We believe that interventional treatments such as surgery, interventional EUS, and endoscopic transpapillary drainage, are valid but should not always be the first choice for AIPassociated PC because PCs were effectively treated in in many patients without a history of CST were effectively treated with CST alone, and this treatment led to the disappearance of the cyst. However, although most of the cystic lesions associated with AIP have been reported as pseudocysts or retention cysts (18), there are some reports describing an association between AIP and neoplastic lesions, such as intraductal papillary mucinous neoplasms, (30) and pan- 
creatic cancer (19). Matsubayashi et al. (19) reported on cases of AIP-associated atypical PCs, such as multilocular cysts or cysts without an obvious corticosteroid response, accompanied by pancreatic ductal carcinoma. Furthermore, some cohort studies showed the development of pancreatic carcinoma (PC) in $2.1-4.8 \%$ (31-33) of cases of AIP during 4.1 (33)-6.1 (31) years of follow-up. Ikeura et al. (32) and Gupta et al. (33) concluded that the risk of PC in patients with AIP was elevated to a similar level to that observed in patients with ordinary chronic pancreatitis. Thus, the resection of cystic lesions should be considered in cases in which malignant lesions are strongly suspected based on diagnostic imaging.

On the other hand, EUS-guided drainage, which has become a standard therapy for pancreatic cystic lesions worldwide (34-39), is considered to be a minimally invasive therapy and to be more suitable than surgical treatment. Although pancreatic cystic lesions (e.g., PC and walled-off necrosis after ordinary acute pancreatitis) are treated by various EUS-guided drainage techniques (e.g., interventional EUS plus the single transluminal gateway technique (SGT), the multiple transluminal gateway technique, and single transluminal gateway transcystic multiple drainage with or without direct endoscopic necrosectomy) (40-42), we believe that SGT alone with the placement of a plastic stent is sufficient treatment for AIP-associated PC AIP because most cases show a unilocular morphology and no necrotic tissues. On the other hand, ESPD using a BFMS for pseudocyst and walled-off necrosis has recently been reported as a useful alternative to using 1 or more plastic stents or a conventional self-expandable biliary metal stent $(43,44)$. A BFMS may provide better drainage due to its large diameter and the use of a BFMS reduces the procedure time and technical complexity because of the ease of placement. Furthermore Mukai et al. showed that there were no significant differences in the rates of adverse events between patients who received BFMSs and those who received plastic stents (45). Thus, when a patient with AIP develops a PC with hemorrhage, SGT using a BFMS might be superior to SGT using a plastic stent because a plastic stent has a risk of stent obstruction with a clot. However, the BFMS should be endoscopically retrieved soon after the size of the cavity has decreased because the effects of long-term metal stent placement have not been fully elucidated. With regard to nasocystic catheters, Siddiqui et al. reported that in the case of AIPassociated PC, nasocystic catheters should be placed for irrigation and to facilitate the prompt improvement of the infected WON (46). Thus, if possible, a nasocystic catheter should have been placed in the remnant cyst in Case 4.

It is also important to analyze the cystic fluid to rule out malignant or premalignant cystic neoplasms when ESPD is performed for pancreatic lesions that are refractory to corticosteroids. Endoscopic transpapillary drainage procedures such as ENPD might be effective, but are associated with a risk of acute pancreatitis and there may also be technical difficulty in inserting a drainage tube because of the diffuse narrowing of the main pancreatic duct, which implies that their indication may be limited.

\section{Conclusion}

ESPD is an effective and minimally invasive treatment for a PC accompanying AIP. We proposed a treatment strategy for a AIP-associated PCs. The first line of treatment is CST, particularly in cases of a PC without a history of CST. However, ESPD can be applied for PCs that are refractory to CST. Surgical treatment should be considered in cases in which malignant lesions are difficult to distinguish.

Informed consent was obtained from all of the patients.

\section{The authors state that they have no Conflict of Interest (COI).}

\section{Financial Support}

This study was approved by our institutional review board (Tokyo Medical University No. 3670).

\section{References}

1. O'Reilly DA, Malde DJ, Duncan T, et al. Review of the diagnosis, classification and management of autoimmune pancreatitis. World J Gastrointest Pathophysiol 5: 71-81, 2014.

2. Ketwaroo G, Sheth S. Autoimmune pancreatitis. Gastroenterol Rep (Oxf) 1: 27-32, 2013.

3. Shimosegawa T, Chari ST, Frulloni L, et al. International consensus diagnostic criteria for autoimmune pancreatitis: guidelines of the International Association of Pancreatology. Pancreas 40: 352358, 2011.

4. Okazaki K, Uchida K, Koyabu M, et al. Recent advances in the concept and diagnosis of autoimmune pancreatitis and IgG4related disease. J Gastroenterol 46: 277-288, 2011.

5. Yoshida K, Toki F, Takeuchi T, et al. Chronic pancreatitis caused by an autoimmune abnormality. Proposal of the concept of autoimmune pancreatitis. Dig Dis Sci 40: 1561-1568, 1995.

6. Muraki T, Hamano H, Ochi Y, et al. Corticosteroid-responsive pancreatic cyst found in autoimmune pancreatitis. J Gastroenterol 40: 761-766, 2005.

7. Welsch T, Kleeff J, Esposito I, et al. Autoimmune pancreatitis associated with a large pancreatic pseudocyst. World J Gastroenterol 12: 5904-5906, 2006.

8. Flack VG, Dixon E. Pseudocyst may be seen in immunoglobulin G4-associated autoimmune pancreatitis. Arch Pathol Lab Med 131: 16, 2007.

9. Kawakami H, Kuwatani M, Shinada K, et al. Autoimmune pancreatitis associated with hemorrhagic pseudocysts: a case report and literature review. Intern Med 47: 603-608, 2008.

10. Sohn JW, Cho CM, Jung MK, et al. A case of autoimmune pancreatitis manifested by a pseudocyst and IgG4-associated cholangitis. Gut Liver 6: 132-135, 2012.

11. Takita M, Itoh $T$, Matsumoto $S$, et al. Autoimmune chronic pancreatitis with IgG4-related pancreatic pseudocyst in a patients undergoing total pancreatectomy followed by autologous islet transplantation a case report. Pancreas 42: 175-177, 2013.

12. Beristain JL, Sabater L, Calatayud A, et al. Autoimmune pancreatitis: inflammatory pseudotumor, multifocal fibrosclerosis, portal hypertension, and long-term outcome. Rev Esp Enferm Dig 100: 652-658, 2013.

13. Hanna MS, Terlevich A, Przemioslo R, Marden P. Autoimmune 
pancreatitis complicated by spontaneous subcapsular splenic haemorrhage. JOP 14: 502-505, 2013.

14. Kitoh H, Kondoh S, Naoki U, et al. Focal autoimmune pancreatitis which discovered for formation of a large pancreatic pseudocyst. Nihon Naika Gakkai Zasshi (J Jpn Soc Int Med) 92: 161-163, 2003 (in Japanese, Abstract in English).

15. Nishimura T, Masaoka T, Suzuki H, et al. Autoimmune pancreatitis with pseudocyst. J Gastroenterol 39: 1005-1010, 2004.

16. Kamisawa T, Anjiki H, Egawa N. Rapid disappearance of a pancreatic cyst after steroid therapy in a patient with autoimmune pancreatitis. Clin Gastroenterol Hepatol 6: e33-e34, 2008.

17. Nishimura N, Tamada K, Wada $S$, et al. Autoimmune pancreatitis associated with a large pancreatic pseudocyst that disappeared after corticosteroid therapy: a case report and literature review. Clin J Gastroenterol 2: 199-203, 2009.

18. Kubota K, Fujita Y, Sato T, et al. Autoimmune pancreatitis associated with pancreatic cyst: how can we manage it? J Hepatobiliary Pancreat Sci 21: 902-910, 2014.

19. Matsubayashi H, Iwai T, Matsui T, et al. Pancreatic cystic lesions with atypical steroid response should be carefully managed in cases of autoimmune pancreatitis. J Gastroenterol Hepatol 31: 270-276, 2016.

20. Chang KA, Kim TN, Lee SH. Autoimmune pancreatitis complicated by an infected pseudocyst. Clin J Gastroenterol 3: 168-173, 2010.

21. Matsubayashi H, Matsui $T$, Ono $H$. Pancreatic duct drainage for the treatment of a huge pancreatic cyst associated with autoimmune pancreatitis. Clin Gastroenterol Hepatol 13: e151-e152, 2015.

22. Clinical Diagnostic Criteria for Autoimmune Pancreatitis 2011 (Proposal) (The Japan Pancreas Society, the Ministry of Health and Welfare Investigation Research Team for Intractable Pancreatic Disease). Suizo 27: 17-25, 2012 (in Japanese, abstract in English).

23. Okamoto T, Niwakawa M, Yasuoka T, et al. Autoimmune pancreatitis with pseudocyst. A case report. Suizo 18: 228-234, 2003 (in Japanese, Abstract in English).

24. Gompertz M, Morales C, Aldana H, et al. Cystic lesions in autoimmune pancreatitis. Case Rep Gastroenterol 9: 366-374, 2015.

25. Maruyama M, Arakura N, Ozaki Y, et al. Risk factors for pancreatic stone formation in autoimmune pancreatitis over a long-term course. J Gastroenterol 47: 553-560, 2012.

26. Kamisawa T, Shimosegawa T, Okazaki K, et al. Standard steroid treatment for autoimmune pancreatitis. Gut 58: 1504-1507, 2009.

27. Ishikawa $\mathrm{T}$, Itoh $\mathrm{A}$, Kawashima $\mathrm{H}$, et al. Peripancreatic vascular involvements of autoimmune pancreatitis. J Gastroenterol Hepatol 27: 1790-1795, 2012.

28. Nakaji S, Hirata N, Fujii H, et al. A case of focal autoimmune pancreatitis (AIP) mimicking an intraductal papillary mucinous neoplasm (IPMN). Clin J Gastroenterol 6: 329-333, 2013.

29. Xu XB, Wu YS, Wang WL, et al. Autoimmune pancreatitis associated with a pancreatic pseudocyst treated by distal pancreatectomy with splenectomy: case report. World J Surg Oncol 12: 359, 2014.

30. Urata $T$, Naito $Y$, Izumi $Y$, et al. Localized type 1 autoimmune pancreatitis superimposed upon preexisting intraductal papillary mucinous neoplasms. World J Gastroenterol 19: 9127-9132, 2013.

31. Hirano K, Tada M, Sasahira N, et al. Incidence of malignancies in patients with IgG4-related disease. Intern Med 53: 171-176, 2014.

32. Ikeura T, Miyoshi H, Uchida K, et al. Relationship between auto- immune pancreatitis and pancreatic cancer: a single-center experience. Pancreatology 14: 373-379, 2014.

33. Gupta R, Khosroshahi A, Shinagare S, et al. Does autoimmune pancreatitis increase the risk of pancreatic carcinoma? A retrospective analysis of pancreatic resections. Pancreas 42: 506-510, 2013.

34. Binmoeller KF, Seifert H, Walter A, et al. Transpapillary and transmural drainage of pancreatic pseudocysts. Gastrointest Endosc 42: 219-224, 1995.

35. Giovannini M, Bernardini D, Seitz JF. Cystogastrostomy entirely performed under endosonography guidance for pancreatic pseudocyst: results in six patients. Gastrointest Endosc 48: 200-203, 1998.

36. Park DH, Lee SS, Moon SH, et al. Endoscopic ultrasound-guided versus conventional transmural drainage for pancreatic pseudocysts: a prospective randomized trial. Endoscopy 41: 842-848, 2009.

37. Varadarajulu S, Christein JD, Tamhane A, et al. Prospective randomized trial comparing EUS and EGD for transmural drainage of pancreatic pseudocysts (with videos). Gastrointest Endosc 68: 1102-1111, 2008.

38. Baron TH, Harewood GC, Morgan DE, et al. Outcome differences after endoscopic drainage of pancreatic necrosis, acute pancreatic pseudocysts, and chronic pancreatic pseudocysts. Gastrointest Endosc 56: 7-17, 2002.

39. Baron TH, Thaggard WG, Morgan DE, et al. Endoscopic therapy for organized pancreatic necrosis. Gastroenterology 111: 755-764, 1996.

40. Varadarajulu S, Phadnis MA, Christein JD, et al. Multiple transluminal gateway technique for EUS-guided drainage of symptomatic wall-off pancreatic necrosis. Gastrointest Endosc 74: 74-80, 2011.

41. Mukai S, Itoi T, Sofuni A, et al. Novel single transluminal gateway transcystic multiple drainages after EUS-guided drainage for complicated multilocular walled-off necrosis (with videos). Gastrointest Endosc 79: 531-535, 2014.

42. Mukai S, Itoi T, Sofuni A, et al. Expanding endoscopic interventions for pancreatic pseudocyst and walled-off necrosis. J Gastroenterol 50: 211-220, 2015.

43. Itoi T, Binmoeller KF, Shah J, et al. Clinical evaluation of a novel lumenapposing metal stent for endosonography-guided pancreatic pseudocyst and gallbladder drainage (with video). Gastrointest Endosc 75: 870-876, 2012.

44. Mukai S, Itoi T, Tsuchiya T, et al. Prospective evaluation of a new biflanged metal stent for the treatment of pancreatic fluid collections (with videos). Gastrointest Endosc 86: 203-207, 2017.

45. Mukai S, Itoi T, Baron TH, et al. Endoscopic ultrasound-guided placement of plastic vs. biflanged metal stents for therapy of walled-off necrosis: a retrospective single-center series. Endoscopy 47: 47-55, 2015.

46. Siddiqui AA, Dewitt JM, Strongin A, et al. Outcomes of EUSguided drainage of debris-containing pancreatic pseudocysts by using combined endoprosthesis and a nasocystic drain. Gastrointest Endosc 78: 589-595, 2013.

The Internal Medicine is an Open Access article distributed under the Creative Commons Attribution-NonCommercial-NoDerivatives 4.0 International License. To view the details of this license, please visit (https://creativecommons.org/licenses/ by-nc-nd/4.0/).

(C) 2018 The Japanese Society of Internal Medicine Intern Med 57: 1523-1531, 2018 